\title{
Autophagy regulating kinases as potential therapeutic targets for age-related macular degeneration
}

Age-related macular degeneration (AMD) is the leading cause of central vision loss in the elderly in the developed countries. The number of AMD patients will double during the next decades due to increasing number of aged people. Chronic oxidative stress, inflammation and accumulation of protein-rich deposits both in the retinal pigment epithelium lysosomes and under the retinal pigment epithelium herald the onset of AMD. The disease can be divided into dry and wet AMD forms. The dry form of the disease is more prevalent accounting for up to $90 \%$ of all cases. Continued intraocular injections are the current treatment strategy to prevent progression of wet AMD. It is a major challenge to develop new drugs that could prevent or at least ease the symptoms of the increasing population of AMD patients. Since AMD pathology is clearly associated with accumulated protein deposits, the autophagy clearance system might represent a potential future therapeutic target for AMD as is thoroughly discussed here.

\section{Age-related macular degeneration as a degenerative eye disease}

Age-related macular degeneration (AMD) is a complex disorder of the eye and the primary cause of irreversible blindness among elderly people in the developed countries [1]. The disease affects the macula enriched with cones in the central area of the retina. AMD has a multifactorial etiology; it results in a progressive loss of fine and colour vision. Globally, 50 million individuals are affected by AMD with the direct cost estimated at US $\$ 255$ billion $[2,3]$. In the USA alone, 1.8 million individuals are afflicted by AMD and the number is estimated to double over the coming decades [4].

AMD is characterized by degeneration of the macular retinal pigment epithelium (RPE) cells, Bruch's membrane and choriocapillaris [5]. AMD etiology is known to be complex, involving both a strong genetic component and several environmental risk factors such as aging, smoking, obesity, arteriosclerosis, hypertension and hypercholesterolemia [6,7]. AMD has a progressive character and it may develop into a dry (non-exudative) or wet (exudative) form. Both forms share certain common clinical features primarily, the degeneration of RPE that is characterized by either hypo- or hyper-pigmentation of the cells and the accumulation of extracellular drusens between the RPE and the inner collagenous layer of the Bruch's membrane (Figure I) [8]. The dry form of the disease is more prevalent and it accounts for up to $90 \%$ of all cases.
In this form, most patients experience a slow, gradual but progressive and inexorable decline in central vision over a period of months or years. Geographic atrophy (GA) is an advanced form of dry AMD being characterized by extensive loss of the RPE, as well as its neighboring photoreceptors (PR) and choriocapillaris and it is associated with severe central visual loss. In some cases, the dry form may be converted into the wet form, in which the visual loss is abrupt, occurring over a time frame of days or weeks. Choroidal neovascularization sprouting into the retina is a diagnostic sign of wet AMD. Central visual loss is due to photoreceptor damage resulting from RPE degeneration, RPE detachment, retinal swelling, accumulation of retinal exudates, choroidal neovascularization, subretinal hemorrhaging and, finally, fibrovascular disciform scarring $[8]$.

To date, no effective cure is available for dry AMD, although antioxidants and omega-fatty acids have been shown to have some preventive properties in certain AMD patient groups [9-11]. VEGFs are potent regulators of angiogenesis in the wet AMD form, and these proteins can be targeted in therapy via their inhibitors: ranibizumab, bevacizumab and VEGF Trap [12]. Large, multicenter, randomized clinical trials have been conducted exploring the safety and efficacy of anti-VEGF treatments [13-16]. However, the need for lifelong intraocular injections are not attractive for patients and, furthermore, these drugs only slow the progression of visual loss. Therefore,

Kai Kaarniranta*1,2,
Anu Kauppinen',
Janusz Blasiak
\& Antero Salminen,
'Department of Ophthalmology,
Institute of Clinical Medicine,
University of Eastern Finland, Kuopio,
Finland
${ }^{2}$ Department of Ophthalmology,
Kuopio University Hospital, Kuopio,
Finland
${ }^{3}$ Department of Molecular Genetics,
University of Lodz, Lodz, Poland
${ }^{4}$ Department of Neurology, Institute
of Clinical Medicine, University of
Eastern Finland, Kuopio, Finland
${ }^{5}$ Department of Neurology, Kuopio
University Hospital, Kuopio, Finland
*Author for correspondence:
Tel.: +358 I7 I72485
Fax: +358 I7 I72486
E-mail: kai.kaarniranta@uef.fi



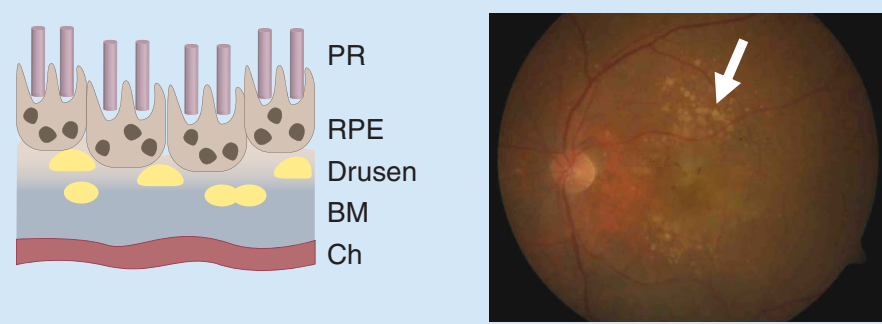

Dry AMD
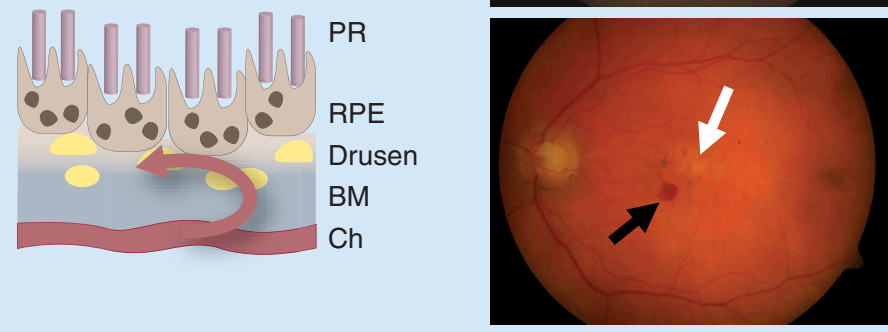

Wet AMD

Figure 1. Schematic and photograph presentation of dry and wet agerelated macular degeneration. Drusens (white arrows) are present in both age-related macular degeneration (AMD) types, but neovascularization (red arrow) from choriocapillaris is a diagnostic sign for wet AMD. Usually, hemorrhages (black arrow) are observed in wet AMD.

AMD: Age-related macular degeneration; BM: Bruch's membrane; Ch: Choriocapillaris; PR: Photoreceptors; RPE: Retinal pigment epithelium.

\section{Key Terms}

\section{Age-related macular} degeneration: Retinal disease that usually affects older adults and results in a loss of fine and color vision in the center of the visual field.

\section{Retinal pigment} epithelium: Pigmented layer of the retina between the neurosensory retina and choroid. It is predominantly retinal pigment epithelium cells that primarily degenerate in age-related macular degeneration.

Autophagy: Catabolic process involving the degradation of a cell's own components through the lysosomal machinery. new drugs are needed to prevent or at least slow development of the most severe AMD forms.

AMD pathogenesis essentially involves a chronic oxidative stress, increased accumulation of lipofuscin in the lysosomes of RPE cells and extracellular drusen formation and chronic inflammation in response to many hereditary and environmental risk factors. The capacity to prevent the accumulation of cellular cytotoxic protein aggregates and the ability to remove them via autophagy decline in aged cells suffering degenerative changes, particularly in postmitotic RPE cells. This article examines autophagy as a possible therapeutic target, that is, finding ways to enhance aggregate clearance and prevent RPE cell degeneration and AMD development.

\section{Central role of RPE in AMD pathogenesis}

Degeneration of the RPE cells is one of the most important hallmarks of AMD (Figure I) [17]. Clinically, it can be observed by pigment dispersion, accumulation of intracellular autofluorescent lysosomal lipofuscin and extracellular drusen deposits. In postmitotic cells such as the RPE cells, the increased oxidative stress during the aging process may be lethal [8,18-22]. The RPE cells constitute a polygonal monolayer between the choroidal capillaries and the rod and cones in the neurosensory retina. They have many important and crucial functions such as transport of metabolites and nutrients between photoreceptors and choriocapillaris, absorption of light energy, production of growth factors for photoreceptors, regulation of homeostasis of the ionic environment, phagocytosis of the retinal outer segments, regulation of the vitamin A-based visual cycle and the creation of the blood-retinal barrier [23].

Constant oxidative stress is a well-known inducer of the protein misfolding and aggregation and this phenomenon is considered to underlie the development of many age-related degenerative diseases including AMD [7]. Ultimately, the pathogenesis of AMD is strongly associated with oxidative stress, resulting in RPE cell damage and degeneration due to the presence of reactive oxygen species (ROS) [8,22,24]. The macula is a region with a high metabolic rate, continuously occurring redox reactions and a high partial pressure due to the adjacent layer of choriocapillaris [25]. High levels of polyunsaturated fatty acid lipids derived from retinal outer segments and the extensive exposure to light increase retinal oxidative stress. In young RPE cells, there are many functional protective mechanisms including a molecular chaperone repair system, antioxidant production and active proteolytic machines, particularly those participating in autophagy [8]. However, in aged RPE cells, the constant exposure to photo-oxidation-generated ROS and phagocytosis of the lipid-rich outer segments expose RPE cells to exceptionally high oxidative stress leading to impaired proteolysis and lipofuscin accumulation in lysosomes $[7,8,26]$. The routine phagocytosis of consumed photoreceptor outer segments and their degradation in lysosomes are two of the critical maintenance functions performed by RPE in order to preserve vision. Lipofuscin includes the fluorophore $N$-retinylidene- $N$-retinylethanolamine, which is a compound that disturbs lysosome membrane stability and inhibits mitochondrial respiration. Subsequently, it is able to promote the unfolding of intracellular proteins [27-30]. In addition to protein damage and aggregation, the amount of oxidized phospholipids is known to be elevated in the macula region of AMD patients [31,32]. These findings highlight the important role of oxidative stress in the pathogenesis of AMD.

\section{Regulation of autophagy by mTOR}

Autophagy, literally, 'self eating', is an intracellular degradation process that clears long-lived proteins, aggregated proteins, lipid droplets and 
organelles from the cytoplasm in all eukaryotic cells [33]. It is activated as an adaptive response to environmental stress (e.g., nutrient deprivation, hypoxia, oxidative stress, exposure to xenobiotics) to promote cell survival through the recycling of precursors (free fatty acid, amino acids, nucleotides) derived from the degradation of endogenous cellular components (FIGURE 2) $[34,35]$. The autophagy process begins with the formation of isolation membranes called omegasomes that enlarge into phagophores. The phagophores then elongate and engulf a portion of the cytosol to form mature double membrane autophagosomes that can engulf portions of cytoplasm containing oligomeric protein complexes and organelles. The autophagosomes fuse with lysosomes and their contents are then degraded by lysosomal enzymes. A failure of autophagy in aged postmitotic cells, incuding RPE cells, can result in the accumulation of aggregate-prone proteins, cellular degeneration and, finally, cell death [8,33-36]. Pharmacological induction of autophagy can enhance the clearance of intracytoplasmic aggregate-prone proteins and ameliorate cellular and tissue pathology [37]. Autophagy, generally, can be viewed as a housekeeping mechanism, and it has crucial significance for the maintenance of normal cellular homeostasis [33-35].

Several autophagy-related proteins (Atg) co-ordinate the autophagosome formation that are composed of four subgroups: the Atg/ ULK complex; the cPtdIns3K/VPs34 complex Atg12-Atg5-Atg16L and Atg8/LC3 conjugation system; and conserved Atg9/mAtg9 transmembrane proteins [38-44]. Atg9 regulates the early phase of autophagosome creation apparently by collecting part of the membranes required for membrane extension. In response to inhibition of mTOR (see later), the ULK complex regulates PtdIns3K complex leading to phagophore formation from which autophagosomes are generated. The Atg12-Atg5-Atg16L conjugation system contributes to the phagophore membrane elongation. LC3-I is transformed to the PE-conjugated form (LC-II), which is inserted into the membrane of the autophagosome $[45,46]$. P62/sequestosome protein binds to LC3-II and acts as facilitator of the degradation of ubiquitinated proteins, thereby linking proteasomal and lysosomal clearance systems $[47,48]$. Finally, the created autophagosomes fuse with lysosomes forming autolysosomes after which lysosomal enzymes are released to degrade the engulfed cytoplasmic material.
There is a growing body of evidence suggesting that regulation of the signaling pathways involved in autophagy could well provide novel pharmaceutical therapeutic strategies for the prevention or treatment of human diseases [49,50]. AMD is of particular interest, since autophagy impairment has been shown in the AMD patient material and RPE cell culture models [51-53]. The classical pathway that regulates autophagy acts through the mTOR, a protein kinase that plays a key role as an energy, nutrient, growth factor, stress and redox sensor to increase protein synthesis and to decrease autophagy (FıGure 2) [54,55]. mTOR is a ubiquitously expressed, 289-kDa serine/threonine kinase belonging to the PI3K-related kinase family. The mTOR consists of the central regulatory catalytic core of two functionally distinct multiprotein complexes, mTORC1 and mTORC2. Rheb, Raptor, PRAS40-P, mLST8 and Deptor are regulatory units for mTORC1, while Sin1, Rictor, mLST8 and Protor are the corresponding units for mTORC2 [56]. Rapamycin, an anti-fungal and immunosuppressant agent, binds to its intracellular receptor FKBP12, which becomes attached to mTOR and interferes with its signalling [55]. Recent findings have revealed that the rapamycin-induced mTORC1 inhibition and activation of autophagy can slow the aging process in mice (Figure 2) [56]. At present, there is convincing evidence that the modulation of mTORC1 function may be a potential target for the development of therapeutics for neurodegenerative diseases including AMD [55-59]. Another way to enhance autophagy is in a mTOR-indirect way, that is, the process regulated by inositol-1,4,5-triphosphate levels. The mTOR-independent autophagy induction is targetted by many drugs such as lithium, sodium valproate, carbamazepine, verapamil and trehalose $[55,60]$.

Interestingly, RPE degeneration has been associated with increased sensitivity and enhanced activity of mTORC1 in experimental AMD studies [61,62]. Rapamycin, an inhibitor of the mTORC1, prevents the development of harmful AMD-related aging signs in RPE cells. It has been shown that mTOR regulates the detrimental dedifferentiation and hypertrophy of RPE cells exposed to oxidative stress, while rapamycin treatment can prevent these effects and preserve photoreceptor functions [61]. Moreover, rapamycin has been observed to inhibit choroidal neovascularisation by inhibiting the function of VEGF-A in animal models [62,63]. Co-culture assays of RPE and endothelial cells revealed 


\section{Key Term}

Sirtuins or Sir2 proteins: Group of proteins that have either histone deacetylase or monoribosyltransferase activities.

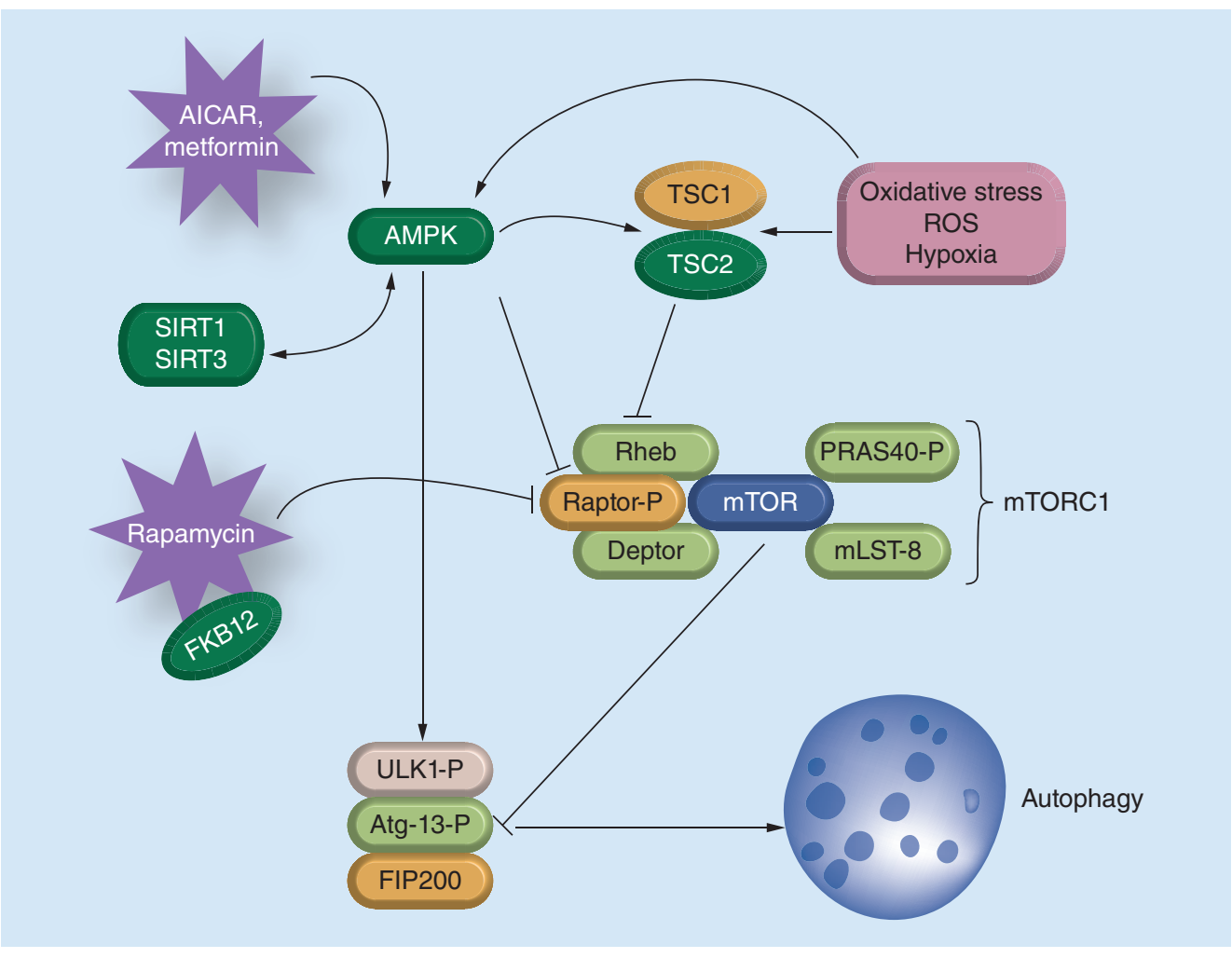

Figure 2. Overview of the autophagy signaling pathways. Oxidative stress, reactive oxygen species production and hypoxia activates AMPK and autophagy clearance. In young cells, this process is working highly effectively, but during aging autophagy clearance is estimated to be suppressed and this leads to a detrimental aggregation of waste products and on to the encountered cellular degeneration in age-related macular degeneration. AICAR, metformin and rapamycin induce autophagy by activating AMPK and/or inactivating mTOR, suppressor of autophagy.

AICAR: 5-aminoimidazole-4-carboxamide ribonucleoside. that rapamycin is an effective VEGF inhibitor and it can reduce sprouting of endothelial cells [64]. The expression of mTOR has been shown to be higher in the central part of retinal layer, which might be one explanation account for greater protein aggregation in aging macula $[8,65]$.

\section{Regulation of autophagy by AMPK}

AMPK is a master sensor of energy and redox status that maintains cellular energy homeostasis. In addition, AMPK regulates autophagy, mitochondrial biogenesis and disposal, cell polarity, cell growth and proliferation. The heterotrimeric protein AMPK has $\alpha, \beta$ and $\gamma$ subunits [66]. The $\alpha$ subunit is the catalytic subunit with $\beta$ and the $\gamma$ being the regulatory subunit is; however, in mammals, each subunit has many subtypes that are expressed at different intensities depending on the tissue [67]. For example, the catalytic $\alpha$ subunit comprises the $\alpha 1$ and $\alpha 2$ isoforms, $\beta$ subunit has the $\beta 1$ and $\beta 2$ isoforms, and $\gamma$ subunit has $\gamma 1, \gamma 2$ and $\gamma 3$ isoforms. The $\alpha$ subunit of AMPK is phosphorylated by several upstream kinases, such as CaMKK $\beta$, LKB1 and TAK1 [68-70]. The $\beta$ subunit provides myristoyl- and carbohydratebinding sites and gathers $\alpha$ and $\gamma$ subunits together [71,72]. Interestingly, degraded oligosaccharides, compounds that are estimated to be involved in RPE aging and AMD development, can suppress AMPK activity $[73,74]$. The $\gamma$ subunit of AMPK is a central sensor to detecting the AMP:ATP ratio in cells [67].

As discussed previously, autophagy is switched off by TORC1. AMPK directly phosphorylates multiple components in the mTORC1 pathway. For instance, AMPK inhibits mTORC1 through phosphorylation of TSC2 and Raptor (FIGURE 2) [55]. Activation of the ULK1 and 2 is the final step prior to autophagosome formation. It has been recently shown that ULK1 and ULK2 form stable complexes with AMPK [75] and ULK1 is a phosphorylation target at multiple sites for AMPK [76,77]. 
AMPK is classically activated by energy depletion and hypoxia. Moreover, a variety of chemicals including the adenosine analogue 5-aminoimidazole-4-carboxamide ribonucleoside (AICAR), biguanides (metformin and phenfor$\mathrm{min}$ ), glycolysis inhibitors (2-deoxy-D-glucose) and metabolic poisons (oligomycin and carbonyl cyanide 4-(trifluoromethoxy)phenylhydrazone) have been used to investigate the role of AMPK in the regulation of the mTORC1 pathway (FIGURe 2).

Qin and Rodriques [78] showed that 4-hydroxynonenal (HNE)-induced lipid peroxidation could evoke RPE cell dysfunction and thus it decreased cell viability in conjunction with the downregulation of basal activity of AMPK. Interestingly, AMPK $\alpha 1$ sensitized RPE cells to the detrimental effects of lipid peroxidation, while AMPK $\alpha 2$ exerted a protective effect. Thus, selective modulation of AMPK $\alpha$ activity may benefit patients with retinal degeneration associated with RPE cell atrophy. AICAR was observed to protect RPE cells in response to oxidative stress [79]. Moreover, AMPK-induced autophagy protected RPE cells from TRAIL -induced cell death [80].

\section{Regulation of autophagy by sirtuins}

Sirtuins have beneficial effects against age-related diseases. They modulate many major biological pathways, such as stress responses, protein aggregation and inflammatory processes, that are involved in age-related diseases. To date, sirtuins have been extensively studied in the context of the age-related pathologies. They are special focusses of interest in drug development for neurodegenerative diseases. Sirtuins are evolutionarily conserved proteins and their name derives from the Sir2 gene of Saccharomyces cerevisiae [81]. Mammals have seven sirtuins (SIRT1-7) and have usually been categorized as NAD+dependent class III histone deacetylase proteins in the histone deacetylase family [81]. Originally, acetylated histones $\mathrm{H} 1, \mathrm{H} 3$ and $\mathrm{H} 4$ were found to serve as physiological substrates for the sirtuins. Subsequently, several non-histone proteins have also been shown to be modulated by sirtuin enzymes [81]. SIRTs 1, 2, 3 and 5 couple protein substrate deacetylation and $\mathrm{NAD}^{+}$cleavage to produce 2'-O-acetyl-ADP-ribose, which together with its nonenzymatically formed derivative, 3'-O-acetyl-ADP-ribose, is involved in the formation of a transcriptionally silent and functionally heterochromatic state [81,82]. SIRT 4 and 6 catalyze the ADP-ribosylation of proteins, although SIRT6 can also act as a deacetylase [81,83,84].
Moreover, along with deacetylation [85], SIRT5 is also capable of modulating protein functions via demalonylation and desuccinylation [86,87].

SIRT1 is the best-studied mammalian sirtuin [88,89]. By deacetylating transcription factors, cofactors, chromatin proteins and the components of the DNA repair machinery, SIRT1 can regulate many significant cellular functions that also play a role in several metabolic, inflammatory, cardiovascular and neurodegenerative diseases, as well as in cancer [81,90]. SIRT1 is well known for its role in increasing the longevity caused by caloric restriction [91]. Since it can deacetylate several crucial transcription factors and co-factors, such as $\mathrm{p} 53$, FoxO proteins, and $\mathrm{NF}-\kappa \mathrm{B}$, it regulates several factors involved in cell's stress resistance and housekeeping [89,91].

In conjunction with SIRT1, 5'AMPAMPK is another enzyme sensing effectively diminished cellular energy states as discussed previously. In order to restore the energy balance, AMPK activates many catabolic processes to generate ATP and it also downregulates the ATP-consuming anabolic processes that are not urgently needed for survival [92,93]. It has become evident that SIRT1 and AMPK are able to stimulate each other (FIGURE 2). When there is a reduced energy state, LKB1 plays a principal role in the phosphorylation and, thus, in the activation of AMPK [94]. AMPK has been reported to activate SIRT1 by increasing the level of the SIRT1 substrate, $\mathrm{NAD}^{+}$[95]. This may be mediated by Nampt, which catalyzes the conversion of nicotinamide to $\mathrm{NAD}^{+}$[95]. On the other hand, it has been shown that SIRT1 can activate AMPK by deacetylating its regulator protein LKB1 [96]. As an example of their shared targets, SIRT1 by deacetylating, and AMPK by phosphorylating activate the transcription cofactor PGC-1 $\alpha$, which promotes the mitochondrial biogenesis and function [97,98].

Recently, more information has started to accumulate also about other sirtuins. The mitochondrial SIRT3 protein has proved to be another sirtuin that can be activated by diet and exercise [99]. In addition to the CREB transcription factor, SIRT3 activates PGC- $1 \alpha$ through AMPK [99-101]. With the help of ERR- $\alpha$, PGC-1 $\alpha$ is also capable of activating SIRT3 [102,103]. SIRT3 activates AMPK by deacetylating LKB1 [104] or by modulating the AMP/ATP ratio [99]. Factors mediating the AMP/ATP regulation have been postulated to include ATP synthase (mitochondrial F1 complex) $\alpha / \beta$ subunits, UQCRH protein and AceCS2 [105,106]. Similar to SIRT1, SIRT3 also deacetylates the transcription factor FoxO3, 
which activates antioxidant genes and, thereby, reduces the intracellular levels of ROS [107]. Moreover, SIRT1 and SIRT3 are able to deacetylate the tumor suppressor protein p53 [108,109]. Hyperacetylation of $\mathrm{p} 53$ induces growth arrest and apoptosis in cells [105], whereas deacetylation promotes cell cycle-dependent autophagocytosis $[110,111]$. Mutual interactions with AMPK, PGC- $1 \alpha$ and other intracellular molecules connect sirtuins into an interesting ensemble, which increases longevity and cell survival via several signaling pathways including those associated with the induction of autophagy. Therefore, they might represent in a target for drugs to treat neurodegenerative diseases including AMD.

\section{Future perspective}

AMD is the most important cause of blindness in individuals over 60 in western countries. AMD is a multifactorial disease resulting from a complex interaction of genetic and environmental factors, age is the only widely recognized risk factor. The socio-economic impact of the disease is enormous, if we take into account the high cost of the available antiangiogenic therapy, the numerous medical visits that it requires and suffering imposes on the patients. One major public health challenge is to find some kind of effective primary preventive measure to combat AMD or to develop more effective drugs so that dry AMD patients could also be treated. To date, there are only recommendations for modifying the risk factors of AMD and only wet AMD patients can be treated and even then only with repeated intravitreal injections that merely slow progression of disease. In recent years, data on mechanisms that regulate autophagy have enormously increased and dysfunctions in these processes are certainly one central component of many neurodegenerative diseases including AMD. However, at the same time, it has been appreciated that autophagy might represent an important therapy target. Particularly, autophagy-regulating kinases AMPK and mTOR both interesting in themselves and as promising targets for preventing RPE cell degeneration and AMD development.

\section{Acknowledgements}

The authors thank E MacDonald for checking the language.

\section{Financial \& competing interest disclosure}

This work was supported by the Academy of Finland, the EVO grant 5503726, the Finnish Cultural Foundation and its North Savo Fund, the Finnish Eye Foundation, Finnish Funding Agency for Technology and Innovation, and the Päivikki and Sakari Sohlberg Foundation. The authors have no other relevant affliations or financial involvement with any organization or entity with a financial interest in or financial conflict with the subject matter or materials discussed in the manuscript apart from those disclosed.

No writing assistance was utilized in the production of this manuscript.

\section{Executive summary}

\section{Age-related macular degeneration as a degenerative eye disease}

- Age-related macular degeneration (AMD) is the most common reason for central vision loss in the elderly in the developed countries.

- The number of patients will double during the next decades.

- The disease is categorized into dry and wet AMD forms.

- Only wet AMD patients can be treated but these only account for approximately $10 \%$ of all AMD cases.

\section{Central role of retinal pigment epithelium in AMD pathogenesis}

- Retinal pigment epithelium degeneration is a primary sign of AMD development.

- Lysosomal lipofuscin accumulation reflects the AMD severity level.

- Extracellular drusens are hallmarks of AMD development.

\section{Regulation of autophagy by mTOR}

- Rapamycin inhibits mTOR and activates autophagy.

- Rapamycin prevent detrimental AMD signs in experimental models.

\section{Regulation of autophagy by AMPK}

- 5-aminoimidazole-4-carboxamide ribonucleoside induces AMPK and autophagy.

- 5-aminoimidazole-4-carboxamide ribonucleoside protect retinal pigment epithelium cells from oxidative stress.

\section{Regulation of autophagy by Sirtuins}

- SIRT1 and SIRT3 interact with AMPK and regulates autophagy.

Sirtuins are longevity factors. 


\section{References}

Papers of special note have been highlighted as:

- of interest

-" of considerable interest

1 Pascolini D, Mariotti SP, Pokharel GP et al. Global update of available data on visual impairment: a compilation of population based prevalence studies. Ophthalmic. Epidemiol. 11(2), 67-115 (2004).

2 Gordois A, Pezzullo L, Cutler H et al. An estimation of the worldwide economic and health burden of visual impairment. Global Public Health 7(5), 465-481 (2012).

3 Gehrs KM, Anderson DH, Johnson LV et al. Age related macular degeneration. Emerging pathogenetic and therapeutic concepts. Ann. Med. 38(7), 450-471 (2006).

- Extensive review for age-related macular degeneration (AMD) pathogenesis.

4 Friedman DS, O'Colmain BJ, Munoz B et al. Prevalence of age related macular degeneration in the United States. Arch. Ophthalmol. 122(4), 564-572 (2004).

5 Jager RD, Mieler WF, Miller JW. Age related macular degeneration. N. Engl. J. Med. 358(24), 2606-2617 (2008).

6 de Jong PT. Age related macular degeneration. N. Engl. J. Med. 355(14), 1474-1485 (2006).

7 Kaarniranta K, Salminen A, Haapasalo A et al. Age-related macular degeneration (AMD): Alzheimer's disease in the eye? J. Alzheimers Dis. 24(4), 615-631 (2011).

8 Kaarniranta K, Salminen A, Eskelinen EL et al. Heat shock proteins as gatekeepers of proteolytic pathways - implications for age-related macular degeneration (AMD). Age. Res. Rev. 8, 128-139 (2009).

- Review of heat shock proteins, proteasomes, and lysosomes and autophagic processes in retinal pigment epithelium (RPE) cell proteolysis and how these might be involved in development of AMD.

9 SanGiovanni JP, Chew EY, Agrón E et al. The relationship of dietary omega-3 long-chain polyunsaturated fatty acid intake with incident age-related macular degeneration: AREDS report no. 23. Arch. Ophthalmol. 126, 1274-1279 (2008).

10 Sin HP, Liu DT, Lam DS. Lifestyle modification, nutritional and vitamins supplements for age-related macular degeneration. Acta Ophthalmol. doi:10.1111/j.1755-3768.2011.02357.x (2012) (Epub ahead of print).

11 Kaarniranta K, Salminen A. NF-kappaB signaling as a putative target for omega-3 metabolites in the prevention of age-related macular degeneration (AMD). Exp. Gerontol. 44(11), 685-688 (2009).

12 Veritti D, Sarao V, Lanzetta P. Neovascular age-related macular degeneration. Ophthalmologica 227(Suppl. 1), 11-20 (2012).

13 CATT Research Group, Martin DF, Maguire MG et al. Ranibizumab and bevacizumab for neovascular age-related macular degeneration. N. Engl. J. Med. 364(20), 1897-1908 (2011).

14 Rosenfeld PJ, Brown DM, Heier JS et al. Ranibizumab for neovascular age-related macular degeneration. N. Engl. J. Med. 355(14), 1419-1431 (2006).

15 Heier JS, Boyer D, Nguyen QD et al. The 1-year results of CLEAR-IT 2, a phase 2 study of vascular endothelial growth factor trap-eye dosed as-needed after 12-week fixed dosing. Ophthalmology 118(6), 1098-1106 (2011).

16 The IVAN Study Investigators Writing Committee. Ranibizumab versus bevacizumab to treat neovascular age-related macular degeneration: one-year findings from the IVAN randomized trial. Ophthalmology 119(7), 1399-1411 (2012).

17 Algvere PV, Seregard S. Age-related maculopathy: pathogenetic features and new treatment modalities. Acta Ophthalmol. Scand. 80, 136-143 (2002).

18 Jarrett SG, Boulton ME. Antioxidant up-regulation and increased nuclear DNA protection play key roles in adaptation to oxidative stress in epithelial cells. Free Radic. Biol. Med. 38(10), 1382-1391 (2005).

19 Kaarniranta K, Ryhänen T, Karjalainen HM et al. Geldanamycin increases 4-hydroxynonenal (HNE)-induced cell death in human retinal pigment epithelial cells. Neurosci. Lett. 382(1-2), 185-190 (2005).

20 Kauppinen A, Niskanen H, Suuronen T, Kinnunen K, Salminen A, Kaarniranta K. Oxidative stress activates NLRP3 inflammasomes in ARPE-19 cellsImplications for age-related macular degeneration (AMD). Immunol. Lett. 147(1-2), 29-33 (2012).

21 Thampi P, Rao HV, Mitter SK et al. The 5HT1a receptor agonist 8-Oh DPAT induces protection from lipofuscin accumulation and oxidative stress in the retinal pigment epithelium. PLoS ONE 7(4), e34468 (2012).

- $\quad 5-\mathrm{HT}\left({ }_{1 \mathrm{~A}}\right)$ receptor agonists may have potential as therapeutic agents in the treatment of retinal degenerative disease as it prevents lipofuscin accumulation and protect the retina from oxidative damage and mitochondrial dysfunction.

22 Klettner A. Oxidative stress induced cellular signaling in RPE cells. Front. Biosci. 1(4), 392-411 (2012).
23 Kaarniranta K, Hyttinen JMT, Ryhänen T et al. Mechanisms of protein aggregation in the retinal pigment epithelial cells. Front. Biosci. 1(2), 1374-1384 (2010).

24 Blasiak J, Szaflik JP. DNA damage and repair in age-related macular degeneration. Front. Biosci. 1(16), 1291-1301 (2011).

25 Roth F, Bindewald A, Holz FG. Key pathologic pathways in age-related macular disease. Graefes Arch. Clin. Exp. Ophthalmol. 242(8), 710-716 (2004).

26 Algvere PV, Marshall J, Seregard S. Age-related maculopathy and the impact of blue light hazard. Acta Ophthalmol. Scand. 84(1), 4-15 (2006).

27 Kaemmerer E, Schutt F, Krohne TU et al. Effects of lipid peroxidation-related protein modifications on RPE lysosomal functions and POS phagocytosis. Invest. Ophthalmol. Vis. Sci. 48(3), 1342-1347 (2007).

28 Vives-Bauza C, Anand M, Shirazi AK et al. The age lipid A2E and mitochondrial dysfunction synergistically impair phagocytosis by retinal pigment epithelial cells. J. Biol. Chem.283(36), 24770-24780 (2008).

29 Finnemann SC, Leung LW, Rodriguez-Boulan E. The lipofuscin component A2E selectively inhibits phagolysosomal degradation of photoreceptor phospholipid by the retinal pigment epithelium. Proc. Natl Acad. Sci. USA 99(6), 3842-3847 (2002).

- Aging RPE accumulates lipofuscin, which includes $N$-retinylidene- $N$ retinylethanolamine as the major autofluorescent component that is involved AMD development.

30 Terman A, Kurz T, Navratil M et al. Mitochondrial turnover and aging of long-lived postmitotic cells: the mitochondrial-lysosomal axis theory of aging, Antiox. Redox. Signal. 12(4), 503-535 (2010).

31 Suzuki M, Kamei M, Itabe H et al. Oxidized phospholipids in the macula increase with age and in eyes with age-related macular degeneration Mol. Vis. 13, 772-778 (2007).

32 Kinnunen PK, Kaarniranta K, Mahalka AK. Protein-oxidized phospholipid interactions in cellular signaling for cell death: From biophysics to clinical correlations. Biochim. Biophys. Acta. 1818(10), 2446-2455 (2012).

33 Yang Z, Klionsky DJ. Eaten alive: a history of macroautophagy. Nat. Cell Biol. 2(9), 814-822 (2010).

34 Yang Z, Klionsky DJ. Mammalian autophagy: core molecular machinery and signalling regulation. Curr. Opin. Cell. Biol. 22(2), 124-131 (2010).

- Highlights the recent advances in identifying and understanding the core molecular 
machinery and signaling pathways that are involved in mammalian autophagy.

35 Mizushima N, Komatsu M. Autophagy: renovation of cells and tissues. Cell 147(4), 728-741 (2011).

- A multidisciplinary review of our current understanding of autophagy's role in metabolic adaptation, intracellular quality control, and renovation during development and differentiation.

36 Salminen A, Kaarniranta K. Regulation of the aging process by autophagy. Trends Mol. Med. 15(5), 217-224 (2009).

37 Harris H, Rubinsztein DC. Control of autophagy as a therapy for neurodegenerative disease. Nat. Rev. Neurol. 8(2), 108-117 (2011).

38 Itakura E, Kishi C, Inoue K et al. Beclin 1 forms two distinct phosphatidylinositol 3-kinase complexes with mammalian Atg14 and UVRAG. Mol. Biol. Cell 19(12), 5360-5372 (2008).

39 Sun Q, Fan W, Chen K et al. Identification of Barkor as a mammalian autophagy-specific factor for Beclin 1 and class III phosphatidylinositol 3-kinase. Proc. Natl Acad. Sci. USA 105(49), 19211-19216 (2008).

40 Ganley IG, Lam DH, Wang J et al. ULK1·ATG13-FIP200 complex mediates mTOR signaling and is essential for autophagy. J. Biol. Chem. 284(18), 12297-12305 (2009).

41 Hosokawa N, Hara T, Kaizuka T et al. Nutrient-dependent $\mathrm{mTORCl}$ association with the ULK1-Atg13-FIP200 complex required for autophagy. Mol. Biol. Cell 20 (7), 1981-1991 (2009).

42 Jung CH, Jun CB, Ro SH et al. ULK-Atg13FIP200 complexes mediate mTOR signaling to the autophagy machinery. Mol. Biol. Cell 20(7), 1992-2003 (2009).

43 Mercer CA, Kaliappan A, Dennis PB. A novel, human Atg13 binding protein, Atg101, interacts with ULK1 and is essential for macroautophagy. Autophagy 5(5), 649-662 (2009).

44 Mari M, Griffith J, Rieter E et al. An Atg9-containing compartment that functions in the early steps of autophagosome biogenesis. J. Cell Biol. 190(6), 1005-1022 (2010).

45 Fujita N, Itoh T, Omori $\mathrm{H}$ et al. The Atg16L complex specifies the site of LC3 lipidation for membrane biogenesis in autophagy. Mol. Biol. Cell 19(5), 2092-2100 (2008).

46 Hanada T, Noda NN, Satomi Y et al. The Atg12-Atg5 conjugate has a novel E3-like activity for protein lipidation in autophagy. J. Biol. Chem. 282(52), 37298-37302 (2007).

47 Pankiv S, Clausen TH, Lamark T et al. p62/ SQSTM1 binds directly to Atg8/LC3 to facilitate degradation of ubiquitinated protein aggregates by autophagy. J. Biol. Chem. 282(33), 24131-24145 (2007).

48 Korolchuk VI, Mansilla A, Menzies FM, Rubinsztein DC. Autophagy inhibition compromises degradation of ubiquitinproteasome pathway substrates. Mol Cell 33(4), 517-527 (2009).

49 Mitter SK, Rao HV, Qi X et al. Autophagy in the retina: a potential role in age-related macular degeneration. Adv. Exp. Med. Biol. 723, 83-90 (2012).

50 Inoki K, Kim J, Guan KL. AMPK and mTOR in cellular energy homeostasis and drug targets. Annu. Rev. Pharmacol. Toxicol. 52, 381-400 (2012).

51 Wang AL, Lukas TJ, Yuan M et al. Autophagy and exosomes in the aged retinal pigment epithelium: possible relevance to drusen formation and age-related macular degeneration. PLoS ONE 24 e4160 (2009).

-. Indication that autophagy and exosomes are closely related in AMD pathogenesis.

52 Ryhänen T, Hyttinen JM, Kopitz J et al. Crosstalk between Hsp70 molecular chaperone, lysosomes and proteasomes in autophagymediated proteolysis in human retinal pigment epithelial cells. J. Cell. Mol. Med. 13(9b), 3616-3631 (2009).

- Interactions of Hsp70, proteasomes and autophagy in the protein turnover of human RPE cells that may open new avenues for understanding degenerative processes in retinal cells.

53 Viiri J, Hyttinen JM, Ryhänen T et al. p62/ sequestosome 1 as a regulator of proteasome inhibitor-induced autophagy in human retinal pigment epithelial cells. Mol. Vis. 16, 1399-1414 (2010).

54 Laplante M, Sabatini DM. mTOR signaling at a glance. Cell Sci. 122(Pt 20), 3589-3594 (2009).

55 Hyttinen JM, Petrovski G, Salminen A et al. 5 -adenosine monophosphate-activated protein kinase--mammalian target of rapamycin axis as therapeutic target for age-related macular degeneration. Rejuvenation Res. 14(6), 651-660 (2011).

56 Wilkinson JE, Burmeister L, Brooks SV et al. Rapamycin slows aging in mice. Aging Cell. 11(4), 675-682 (2012) (2012).

57 Trüe O, Matthias P. Interplay between histone deacetylases and autophagy - from cancer therapy to neurodegeneration. Immunol. Cell Biol. 90(1), 78-84 (2012).

58 Bové J, Martínez-Vicente M, Vila M. Fighting neurodegeneration with rapamycin: mechanistic insights. Nat. Rev. Neurosci. 12(8), 437-452 (2011).
59 Yang DS, Stavrides P, Mohan PS et al. Therapeutic effects of remediating autophagy failure in a mouse model of Alzheimer disease by enhancing lysosomal proteolysis. Autophagy 7(7), 788-789 (2011).

60 Sarkar S, Ravikumar B, Floto RA et al. Rapamycin and mTOR-independent autophagy inducers ameliorate toxicity of polyglutamine-expanded huntingtin and related proteinopathies. Cell Death Differ. 16(1), 46-56 (2009).

61 Zhao C, Yasumura D, Li X et al. mTORmediated dedifferentiation of the retinal pigment epithelium initiates photoreceptor degeneration in mice. J. Clin. Invest. 121, 369-383 (2011).

62 Li Y, Huang D, Xia X, Wang Z et al. CCR3 and choroidal neovascularization. PLoS ONE 6, e17106 (2011).

63 Dejneka NS, Kuroki AM, Fosnot J et al. Systemic rapamycin inhibits retinal and choroidal neovascularizaion in mice. Mol. Vis. 10, 964-972 (2004).

64 Stahl A, Paschek L, Martin G et al. Rapamycin reduces VEGF expression in retinal pigment epithelium (RPE) and inhibits RPE-induced sprouting angiogenesis in vitro. FEBS Lett. 582(20), 3097-3102 (2008).

65 Kokkinopoulos I, Shahabi G, Colman A et al. Mature peripheral RPE cells have an intrinsic capacity to proliferate; a potential regulatory mechanism for age-related cell loss. PLoS ONE 6, e18921 (2011).

66 Hardie DG. AMP-activated protein kinase: an energy sensor that regulates all aspects of cell function. Genes Dev. 25(18), 1895-1908 (2011).

67 Mihaylova MM, Shaw RJ. The AMPK signalling pathway coordinates cell growth, autophagy and metabolism. Nat. Cell Biol. 13(9), 1016-1023 (2011).

68 Momcilovic M, Hong SP, Carlson M. Mammalian TAK1 activates $S n f 1$ protein kinase in yeast and phosphorylates AMPactivated protein kinase in vitro. J. Biol. Chem. 281(35), 25336-25343 (2006).

69 Hawley SA, Selbert MA, Goldstein EG et al. 5-AMP activates the AMP-activated protein kinase cascade, and $\mathrm{Ca}^{2+} /$ calmodulin activates the calmodulin-dependent protein kinase I cascade, via three independent mechanisms. J. Biol. Chem. 270 (45), 27186-27191 (1995).

70 Hong SP, Leiper FC, Woods A et al. Activation of yeast $\mathrm{Snf} 1$ and mammalian AMP-activated protein kinase by upstream kinases. Proc. Natl Acad. Sci. USA 100(15), 8839-8843 (2003).

71 Warden SM, Richardson C, O'Donnell J Jr et al. Post-translational modifications of the $\beta-1$ subunit of AMP-activated protein kinase 
affect enzyme activity and cellular localization. Biochem. J. 354(Pt 2), 275-283 (2001).

72 Oakhill JS, Chen ZP, Scott JW et al. $\beta$-Subunit myristoylation is the gatekeeper for initiating metabolic stress sensing by AMP-activated protein kinase (AMPK). Proc. Natl Acad. Sci. USA 107(45), 19237-19241 (2010).

73 McBride A, Ghilagaber S, Nikolaev A et al. The glycogen-binding domain on the AMPK $\beta$ subunit allows the kinase to act as a glycogen sensor. Cell Metab. 9(1), 23-34 (2009).

74 Kaarniranta K, Salminen A. Age-related macular degeneration: activation of innate immunity system via pattern recognition receptors. J. Mol. Med. 87(2), 117-123 (2009).

75 Behrends C, Sowa ME, Gygi SP et al. Network organization of the human autophagy system. Nature 466(7302), 68-76 (2010).

76 Egan DF, Shackelford DB, Mihaylova MM et al. Phosphorylation of ULK1 (hATG1) by AMP-activated protein kinase connects energy sensing to mitophagy. Science 331(6016), 456-461 (2011).

77 Kim J, Kundu M, Viollet B et al. AMPK and mTOR regulate autophagy through direct phosphorylation of Ulk1. Nat. Cell Biol. 13(2), 132-141 (2011).

78 Qin S, Rodrigues GA. Differential roles of AMPK $\alpha 1$ and AMPK $\alpha 2$ in regulating 4-HNE-induced RPE cell death and permeability. Exp. Eye Res. 91(6), 818-824 (2010).

79 Qin S, De Vries GW. $\alpha 2$ But not $\alpha 1$ AMP-activated protein kinase mediates oxidative stress-induced inhibition of retinal pigment epithelium cell phagocytosis of photoreceptor outer segments. J. Biol. Chem. 283(11), 6744-6751 (2008).

80 Herrero-Martín G, Høyer-Hansen M, García-García C et al. TAK1 activates AMPK-dependent cytoprotective autophagy in TRAIL-treated epithelial cells. EMBO J. 28(6), 677-685 (2009).

81 Yamamoto H, Schoonjans K, Auwerx J. Sirtuin functions in health and disease. Mol. Endocrinol. 21(8), 1745-1755 (2007).

82 Hoff KG, Wolberger C. Getting a grip on O-acetyl-ADP-ribose. Nat. Struct. Mol. Biol. 12(7), 560-561 (2005).

83 Michishita E, McCord RA, Berber E et al. SIRT6 is a histone H3 lysine 9 deacetylase that modulates telomeric chromatin. Nature 452(7186), 492-496 (2008).

84 Kawahara TL, Michishita E, Adler AS et al. SIRT6 links histone H3 lysine 9 deacetylation to NF-kappaB-dependent gene expression and organismal life span. Cell. 136(1), 62-74 (2009).
85 Nakagawa T, Lomb DJ, Haigis MC et al. SIRT5 Deacetylates carbamoyl phosphate synthetase 1 and regulates the urea cycle. Cell 137(3), 560-570 (2009).

86 Du J, Zhou Y, Su X et al. Sirt5 is a NADdependent protein lysine demalonylase and desuccinylase. Science 334(6057), 806-809. (2011).

87 Peng C, Lu Z, Xie Z et al. The first identification of lysine malonylation substrates and its regulatory enzyme. Mol. Cell. Proteomics. 10(12), M111.012658 (2011).

88 Blander G, Bhimavarapu A, Mammone T et al. SIRT1 promotes differentiation of normal human keratinocytes. J. Invest. Dermatol. 129(1), 41-49 (2009).

89 Lavu S, Boss O, Elliott PJ, Lambert PD. Sirtuins - novel therapeutic targets to treat ageassociated diseases. Nat. Rev. Drug Discov. 7(10), 841-853 (2008).

90 Guarente L. Franklin H. Epstein lecture: sirtuins, aging, and medicine. N. Eng. J. Med. 364(23), 2235-2244 (2009).

91 Wang Y, Liang Y, Vanhoutte PM. SIRT1 and AMPK in regulating mammalian senescence: a critical review and a working model. FEBS Lett. 585(7), 986-994 (2011).

92 Cantó C, Gerhart-Hines Z, Feige JN et al. AMPK regulates energy expenditure by modulating $\mathrm{NAD}^{+}$metabolism and SIRT1 activity. Nature 458(7241), 1056-1060 (2009).

93 Hardie DG, Hawley SA, Scott JW. AMPactivated protein kinase--development of the energy sensor concept. J. Physiol. 574(Pt 1), 7-15 (2006).

94 Beauloye C, Bertrand L, Horman S et al. AMPK activation, a preventive therapeutic target in the transition from cardiac injury to heart failure. Cardiovasc. Res. 90(2), 224-233 (2011).

95 Fulco M, Cen Y, Zhao P et al. Glucose restriction inhibits skeletal myoblast differentiation by activating SIRT1 through AMPK-mediated regulation of Nampt. Dev. Cell 14(5), 661-673 (2008).

96 Lan F, Cacicedo JM, Ruderman N et al. SIRT1 modulation of the acetylation status, cytosolic localization, and activity of LKB1. Possible role in AMP-activated protein kinase activation. J. Biol. Chem. 283(41), 27628-27635 (2008).

97 Nemoto S, Fergusson MM, Finkel T. SIRT1 functionally interacts with the metabolic regulator and transcriptional coactivator PGC-1 $\{\alpha\}$. J. Biol. Chem. $280(16)$, 16456-16460 (2005).

98 Scarpulla RC. Metabolic control of mitochondrial biogenesis through the PGC-1 family regulatory network. Biochim. Biophys. Acta 1813(7), 1269-1278 (2011).
99 Palacios OM, Carmona JJ, Michan S et al. Diet and exercise signals regulate SIRT3 and activate AMPK and PGC-1alpha in skeletal muscle. Aging 1(9), 771-783 (2009).

100 Shi T, Wang F, Stieren E, Tong Q. SIRT3, a mitochondrial sirtuin deacetylase, regulates mitochondrial function and thermogenesis in brown adipocytes. J. Biol. Chem. 280(14), 13560-13567 (2005).

101 Shi T, Fan GQ, Xiao SD. SIRT3 reduces lipid accumulation via AMPK activation in human hepatic cells. J. Dig. Dis. 11(1), 55-62 (2010).

102 Kong X, Wang R, Xue Y et al. Sirtuin 3, a new target of PGC-1alpha, plays an important role in the suppression of ROS and mitochondrial biogenesis. PLoS ONE 5(7), e11707 (2010).

103 Giralt A, Hondares E, Villena JA et al. Peroxisome proliferator-activated receptorgamma coactivator-1alpha controls transcription of the Sirt3 gene, an essential component of the thermogenic brown adipocyte phenotype. J. Biol. Chem. 286(19), 16958-16966 (2011).

104 Pillai VB, Sundaresan NR, Kim G et al. Exogenous NAD blocks cardiac hypertrophic response via activation of the SIRT3-LKB1AMP-activated kinase pathway. J. Biol. Chem. 285(5), 3133-3144 (2010).

105 Law IK, Liu L, Xu A et al. Identification and characterization of proteins interacting with SIRT1 and SIRT3: implications in the anti-aging and metabolic effects of sirtuins. Proteomics 9(9), 2444-2456 (2009).

106 Hallows WC, Lee S, Denu JM. Sirtuins deacetylate and activate mammalian acetyl-CoA synthetases. Proc. Natl Acad. Sci. USA 103(27), 10230-10235 (2006).

107 Sundaresan NR, Gupta M, Kim G et al. Sirt3 blocks the cardiac hypertrophic response by augmenting Foxo3a-dependent antioxidant defense mechanisms in mice. J. Clin. Invest. 119(9), 2758-2771 (2009).

108 Lynch CJ, Shah ZH, Allison SJ et al. SIRT1 undergoes alternative splicing in a novel auto-regulatory loop with p53. PLoS ONE 5(10), e13502 (2010).

109 Salminen A, Kaarniranta K. SIRT1: regulation of longevity via autophagy. Cell Signal. 21(9), 1356-1360 (2009).

110 Tasdemir E, Maiuri MC, Orhon I et al. p53 represses autophagy in a cell cycle-dependent fashion. Cell Cycle 7(19), 3006-3011 (2008).

111 Tavernarakis N, Pasparaki A, Tasdemir E et al. The effects of p53 on whole organism longevity are mediated by autophagy. Autophagy 4(7), 870-873 (2008). 